\title{
Nonlinear dynamics and chaos in systems with discontinuous support
}

\author{
Sandor Divenyi ${ }^{\mathrm{a}}$, Marcelo Amorim Savi ${ }^{\mathrm{a}}$, Luiz Fernando Penna Franca ${ }^{\mathrm{b}}$ and Hans Ingo Weber ${ }^{\mathrm{c}}$ \\ ${ }^{a}$ Department of Mechanical Engineering, Universidade Federal do Rio de Janeiro, COPPE, 21.941.972, Rio de \\ Janeiro, RJ, P.O. Box 68.503, Brazil \\ E-mail: savi@ufrj.br \\ ${ }^{\mathrm{b}}$ CSIRO Petroleum, Drilling Mechanics, Kensington WA 6151, P.O. Box 1130, Bentley WA 6102, Australia \\ ${ }^{\text {c } P o n t i f i ́ c i a ~ U n i v e r s i d a d e ~ C a t o ́ l i c a ~ d o ~ R i o ~ d e ~ J a n e i r o, ~ D e p a r t m e n t ~ o f ~ M e c h a n i c a l ~ E n g i n e e r i n g, ~ 22.453 .900, ~ R i o ~ d e ~}$ \\ Janeiro, RJ, Brazil
}

\begin{abstract}
Non-smooth systems are abundant in nature being usually related to physical systems with dry friction, impact and backlash. These systems operate in different modes, and the transition from one mode to another can often be idealized as an instantaneous or discrete transition. Since the time scale of this transition is much smaller than the scale of the individual modes dynamics, its mathematical modeling can be lead as non-smooth. This contribution uses a smoothened switch model to analyze non-smooth systems. The procedure seems to be effective to deal with this kind of system, presenting advantages for the numerical implementation. As an application of the general formulation, a single-degree of freedom oscillator with discontinuous support is analyzed. System dynamical behavior shows a rich response, presenting dynamical jumps, bifurcations and chaos.
\end{abstract}

Keywords: Nonlinear dynamics, non-smooth systems, bifurcations, chaos, grazing

\section{Introduction}

Non-smooth nonlinearity is abundant in nature being usually related to either the friction phenomenon or the discontinuous characteristics as intermittent contacts of some system components. Therefore, physical systems with dry friction, impact and backlash operate in different modes, and the transition from one mode to another can often be idealized as an instantaneous or discrete transition. Since the time scale of this transition is much smaller than the scale of the individual mode dynamics their modeling results in non-smooth systems.

Non-smooth systems appear in many kinds of engineering systems and also in everyday life. Examples may be mentioned by the stick-slip oscillations of a violin string or grating brakes [4]. Some related phenomena as chatter and squeal causes serious problems in many industrial applications and, in general, these forms of vibrations are undesirable because of their detrimental effects on the operation and performance of mechanical systems. They can cause excessive wear of components, surface damage, fatigue failure and noise [1].

The mathematical modeling and numerical simulations of non-smooth systems present many difficulties, which turn their description unusually complex. Moreover, the dynamical behavior of these systems is complex, presenting a rich response. Therefore, non-smooth systems present scientific as well as technological interests that are motivating different researches related to them.

Literature presents many reports dealing with non-smooth systems, concerning the mathematical modeling, the proposition of proper numerical algorithms to treat these systems, and also experimental approaches employed to verify the obtained results. In all these works, it should be pointed out a complex dynamical response. The idea that non-smooth systems can be considered as continuous in a finite number of continuous subspaces and also that the system parameters do not change in an abrupt manner, inspires some authors to try to describe non-smooth systems 


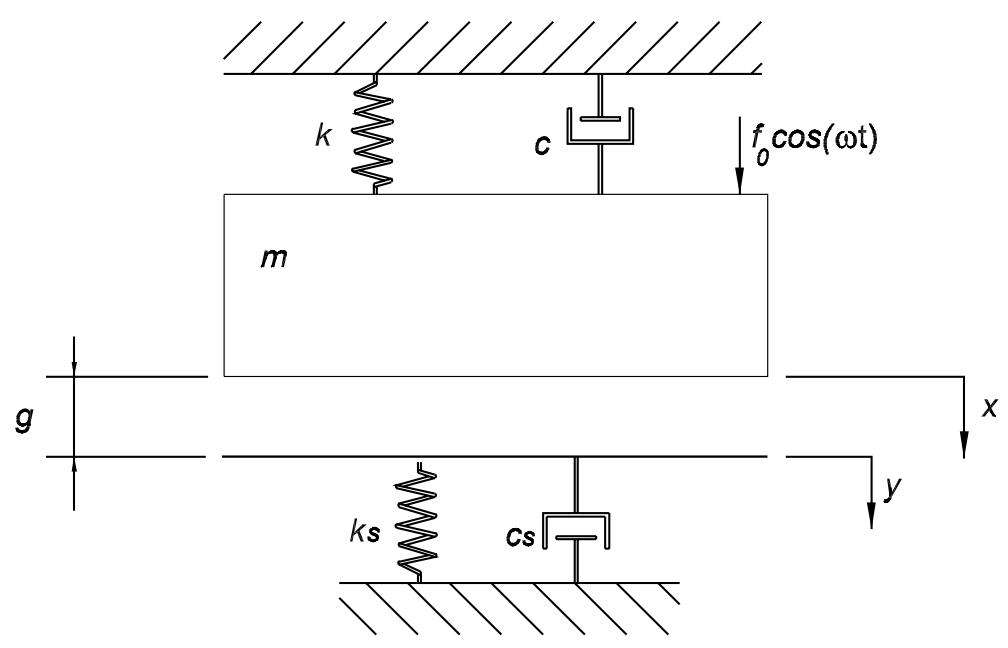

Fig. 1. Non-smooth system with discontinuous support.

by a smoothened form. Wiercigroch [15], Leine [9], Leine et al. [8], Leine and van Campen [5-7] are some efforts where interesting approaches are proposed in order to deal with mathematical discontinuity.

The objective of this contribution is the nonlinear dynamics analysis of non-smooth systems. As an application, a single-degree of freedom system with discontinuous support is of concern. Despite the deceiving simplicity of this problem, its nonlinear dynamics is very rich. The mathematical model uses a smoothened switch model, proposed by Leine [9] on the study of stick-slip vibrations. Basically, the switch model treats non-smooth systems defining different sets of ordinary differential equations. Each set is related to a subspace of the physical system. The innovative idea is the definition of transition regions that governs the dynamical response during the transition from one set to another. Therefore, each subspace has its own ordinary differential equation. Besides, each transition region also has its governing equation, defined in order to smooth the system dynamics. The use of this approach smoothes the discontinuities and allows the use of classical numerical procedures. Numerical investigations of the single-degree of freedom system with a discontinuous support show efficiency and allow one to analyze many aspects related to the non-smooth system dynamics.

\section{Discontinuous support model}

This contribution analyzes the dynamical response of a single-degree of freedom system with discontinuous support, shown in Fig. 1. The oscillator is harmonically excited being composed by a mass $m$, connected with a linear spring with stiffness $k$ and a linear damping with coefficient $c$. Moreover, the support is massless, having a linear spring with stiffness $k_{s}$ and a linear damping with coefficient $c_{s}$. The displacement of the mass is denoted by $x$, relative to the equilibrium position, while the displacement of the support is denoted by $y(y \geqslant 0)$. A gap $g$ defines the distance between the mass and the support. Therefore, the system has two possible modes, represented by a situation where the mass presents contact with the support and other situation when there is no contact. Calling $f_{s}$ as the contact force between the mass and the support, these two situations may be represented as follows:

$$
\left\{\begin{array}{l}
x<g \text { and } f_{s}=0, \text { without contact. } \\
x \geqslant g \text { and } f_{s}=-\left(k_{s} y+c_{s} \dot{y}\right)<0, \text { with contact. }
\end{array}\right.
$$

The support relaxes to the equilibrium state when there is no contact between the mass and the support. By assuming that the relaxation time of the support is much smaller than the time between two contact events, the dynamics of the support can be neglected. This assumption reduces the system dynamics to a second-order differential equation associated with the mass-spring-dashpot dynamics. Therefore, the system governing equations may be defined by two different equations. One for situations without contact and the other with contact: 
$\left\{\begin{array}{l}m \ddot{x}+k x+c \dot{x}=f_{0} \cos (\omega t), \text { without contact. } \\ m \ddot{x}+k x+k_{s}(x-g)+\left(c+c_{s}\right) \dot{x}=f_{0} \cos (\omega t), \text { with contact. }\end{array}\right.$

By defining a non-dimensional variable $X=x / g$ and also,

$$
\omega_{0}^{2}=k / m ; \omega_{s}^{2}=k_{s} / m ; \xi=c / m ; \xi_{s}=c_{s} / m ; F_{0}=f_{0} / m g
$$

the equations of motion are rewritten as follows,

$$
\left\{\begin{array}{l}
\ddot{X}+\omega_{0}^{2} X+\xi \dot{X}=F_{0} \cos (\omega t), \text { without contact. } \\
\ddot{X}+\omega_{0}^{2} X+\omega_{s}^{2}(X-1)+\left(\xi+\xi_{s}\right) \dot{X}=F_{0} \cos (\omega t), \text { with contact. }
\end{array}\right.
$$

According to the Filippov theory [3,9], the state space of a system $\dot{u}=\tilde{f}(u), \quad u=(X, \dot{X})$ may be split into two subspaces $\tilde{\Gamma}_{-}$and $\tilde{\Gamma}_{+}$, separated by a hyper-surface $\tilde{\Sigma}$, which is defined by a scalar indicator function $h(u)$.

$$
\dot{u}=\tilde{f}(u, t)=\left\{\begin{array}{l}
\tilde{f}_{-}, u \in \tilde{\Gamma}_{-} \\
\tilde{f}_{+}, u \in \tilde{\Gamma}_{+}
\end{array}\right.
$$

where the subspaces $\tilde{\Gamma}_{-}$and $\tilde{\Gamma}_{+}$, and the hyper-surface $\tilde{\Sigma}$, are defined as follows:

$$
\begin{aligned}
\tilde{\Gamma}_{-} & =\left\{u \in R^{n} / h(u)<0\right\} \\
\tilde{\Sigma} & =\left\{u \in R^{n} / h(u)=0\right\} \\
\tilde{\Gamma}_{+} & =\left\{u \in R^{n} / h(u)>0\right.
\end{aligned}
$$

The use of Filippov theory in the oscillator with discontinuous support imposes a correct description of the transitions. The contact between the mass and the support occurs if the displacement becomes equal to the gap $g$. On the other hand, the mass loses contact with the support when the contact force vanishes, i.e., if $f_{s}=-\left[k_{s}(x-g)+c_{s} \dot{x}\right]=0$, or in non-dimensional terms, $F_{s}=-\left[\omega_{s}^{2}(X-1)+\xi_{s} \dot{X}\right]=0$. Therefore, there are two subspaces with two different transition situations. The subspaces are defined by two indicator functions:

$$
\begin{aligned}
& h_{\alpha}(x, \dot{x})=X-1 \\
& h_{\beta}(x, \dot{x})=-(X-1)-\frac{\xi_{s}}{\omega_{s}^{2}} \dot{X}
\end{aligned}
$$

Now, it is possible to describe the mass motion, considering that there is no contact with the support if the state vector $u \in \tilde{\Gamma}_{-}$. On the other hand, there is contact between the mass and the support if $u \in \tilde{\Gamma}_{+}$. These situations are represented by:

$$
\begin{aligned}
& \tilde{\Gamma}_{-}=\left\{u \in R^{2} / h_{\alpha}(u)<0 \text { or } h_{\beta}(u)>0\right\} \\
& \tilde{\Gamma}_{+}=\left\{u \in R^{2} / h_{\alpha}(u)>0 \text { and } h_{\beta}(u)<0\right\}
\end{aligned}
$$

The transitions are related to hyper-surface $\tilde{\Sigma}$, which consists of the conjunction of two surfaces $\tilde{\Sigma}_{\alpha}$ and $\tilde{\Sigma}_{\beta}$. The hyper-surface $\tilde{\Sigma}_{\alpha}$ defines the transition from $\tilde{\Gamma}_{-}$to $\tilde{\Gamma}_{+}$, representing situations where the contact is caused when $u$ becomes greater than $g$. On the other hand, the hyper-surface $\tilde{\Sigma}_{\beta}$ defines the transition from $\tilde{\Gamma}_{+}$to $\tilde{\Gamma}_{-}$as the contact is lost when the support force vanishes. Therefore,

$$
\begin{aligned}
& \tilde{\Sigma}_{\alpha}=\left\{u \in R^{2} / h_{\alpha}(u)=0 \text { and } h_{\beta}(u) \leqslant 0\right\} \\
& \tilde{\Sigma}_{\beta}=\left\{u \in R^{2} / h_{\alpha}(u)>0 \text { and } h_{\beta}(u)=0\right\}
\end{aligned}
$$

This non-smooth system may be smoothened redefining the subspaces and the transition hyper-surfaces, assuming that the transition has a linear variation from $\tilde{f}_{-}$to $\tilde{f}_{+}$(or from $\tilde{f}_{+}$to $\left.\tilde{f}_{-}\right)$in a thin space define by a narrow band 

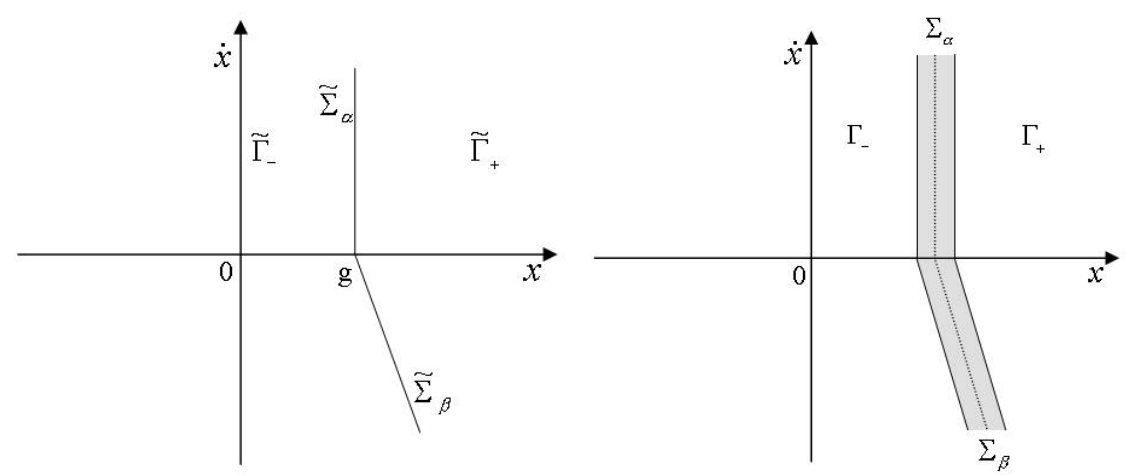

Fig. 2. Subspaces related to the system dynamics.

$\eta=\hat{\eta} / g$ around the hyper-surface of discontinuity. Figure 2 shows either the subspaces or the hyper-surfaces related to the single-degree of freedom dynamics, considering both non-smooth and smoothened systems.

Assuming a transition through a narrow band with thickness $\eta$, system can be written as follows [9],

$$
\dot{u}=f(u, t)=\left\{\begin{array}{l}
f_{-}, u \in \Gamma_{-} \\
f_{\alpha}, u \in \Sigma_{\alpha} \\
f_{\beta}, u \in \Sigma_{\beta} \\
f_{+}, u \in \Gamma_{+}
\end{array}\right.
$$

where,

$$
\begin{aligned}
& f_{-}(u, t)=\left\{\begin{array}{l}
\dot{X} \\
-\omega_{0}^{2} X-\xi \dot{X}+F_{0} \cos (\omega t)
\end{array}\right\} \\
& f_{+}(u, t)=\left\{\begin{array}{l}
\dot{X} \\
-\omega_{0}^{2} X-\omega_{s}^{2}(X-1)-\left(\xi+\xi_{s}\right) \dot{X}+F_{0} \cos (\omega t)
\end{array}\right\}
\end{aligned}
$$

The definition of the transitions associated with hyper-surfaces $\sum_{\alpha}$ and $\sum_{\beta}$, is defined by assuming that,

$$
\begin{aligned}
& f_{\alpha}=(1-q) f_{-}+q f_{+}, q=(X-1+\eta) / 2 \eta \\
& f_{\beta}=(1-q) f_{+}+q f_{-}, q=\left[-X+1+\eta-\left(\xi_{s} / \omega_{s}^{2}\right) \dot{X}\right] / 2 \eta
\end{aligned}
$$

Notice that $0 \leqslant q \leqslant 1$. Under these assumptions, there is a time instant $t_{a}$ where $q=0$, the input limit of region $\sum_{\alpha}$, and also a time instant $t_{b}$ where $q=1$, the output limit of region $\sum_{\alpha}$. The proposed expression considers a linear approximation through the transition region, from $f_{-}\left(t_{a}\right)$ to $f_{+}\left(t_{b}\right)$. In an analogous form, there is a time instant $t_{c}$ where $q=0$, the input limit of region $\sum_{\beta}$, and also a time instant $t_{d}$ where $q=1$, the output limit of region $\sum_{\beta}$. Again, it is assumed a linear approximation through the transition region, from $f_{+}\left(t_{c}\right)$ to $f_{-}\left(t_{d}\right)$. Therefore, the transitions are defined as follows:

$$
\begin{aligned}
& f_{\alpha}=\left\{\begin{array}{l}
\dot{X} \\
-\omega_{0}^{2} X-\left(\xi+\xi_{s}\right) \dot{X}+F_{0} \cos (\omega t)-\frac{1}{2} \omega_{s}^{2}(X-1+\eta)+\xi_{s} \dot{X}_{a}\left(1-\frac{X-1+\eta}{2 \eta}\right)
\end{array}\right\} \\
& f_{\beta}=\left\{\begin{array}{l}
\dot{X} \\
-\omega_{0}^{2} X-\xi \dot{X}+F_{0} \cos (\omega t)-\frac{1}{2} \omega_{s}^{2}(X-1+\eta)-\frac{1}{2} \xi_{s} \dot{X}
\end{array}\right\}
\end{aligned}
$$

The subspaces and transitions hyper-surfaces are now defined by: 
Table 1

System parameters

\begin{tabular}{cccccc}
\hline$\omega_{0}(\mathrm{rad} / \mathrm{s})$ & $\omega_{s}(\mathrm{rad} / \mathrm{s})$ & $\xi\left(\mathrm{s}^{-1}\right)$ & $\xi_{s}\left(\mathrm{~s}^{-1}\right)$ & $F_{0}\left(\mathrm{~s}^{-2}\right)$ & $\omega(\mathrm{rad} / \mathrm{s})$ \\
\hline 4.5 & 38.0 & 1.04 & 0.72 & 144.9 & 5 \\
\hline
\end{tabular}
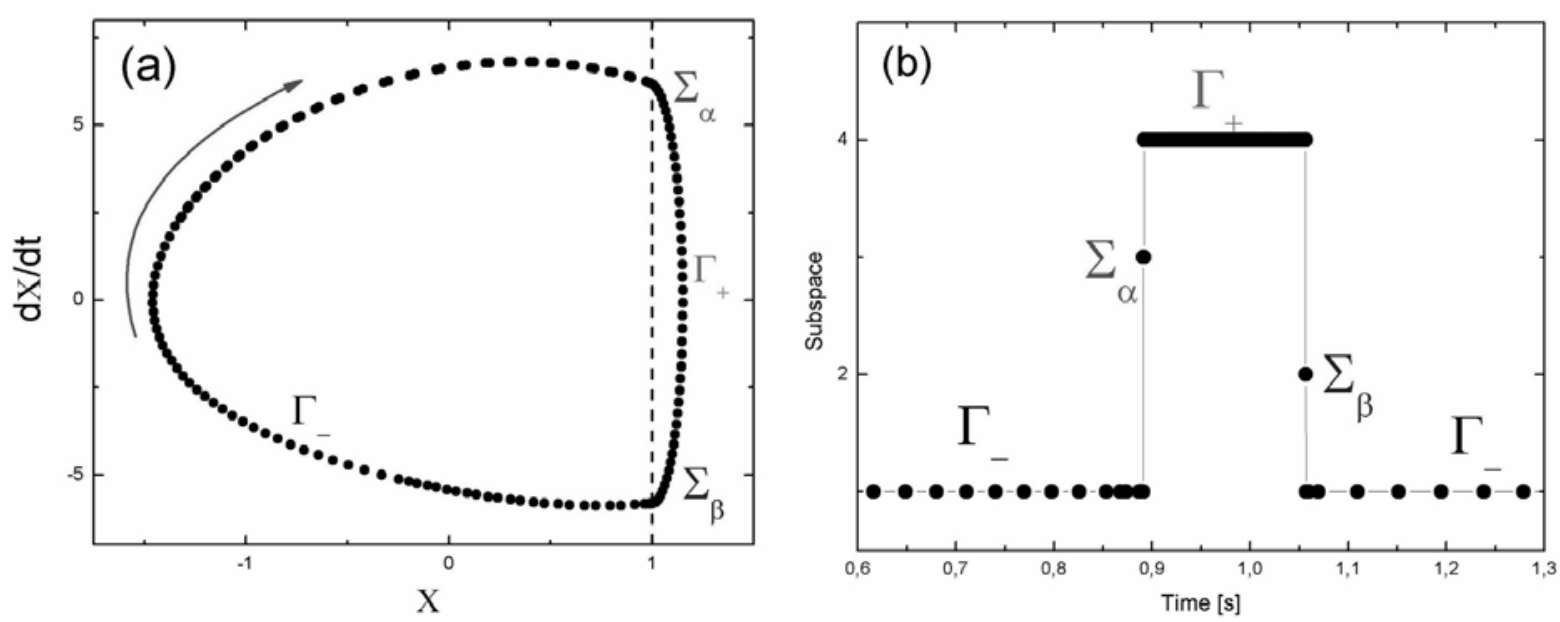

Fig. 3. Analysis of system evolution through the subspaces.

$$
\begin{aligned}
& \Gamma_{-}=\left\{u \in R^{2} / h_{\alpha}(u) \leqslant-\eta \text { or } h_{\beta}(u) \geqslant \eta\right\} \\
& \Gamma_{+}=\left\{u \in R^{2} / h_{\alpha}(u) \geqslant \eta \text { and } h_{\beta}(u) \leqslant-\eta\right\} \\
& \Sigma_{\alpha}=\left\{u \in R^{2} /-\eta<h_{\alpha}(u)<+\eta \text { and } h_{\beta}(u) \leqslant-h_{\alpha}(u)\right\} \\
& \Sigma_{\beta}=\left\{u \in R^{2} / h_{\alpha}(u)>-h_{\beta}(u) \text { and }-\eta<h_{\beta}(u)<+\eta\right\}
\end{aligned}
$$

This approach allows one to deal with non-smooth systems employing a smoothened system. The thickness parameter of the narrow band $\eta$ need to be appropriated chose for each physical problem. This switch model is introduced as an appropriate procedure to perform mathematical modeling allowing an efficient numerical procedure to describe non-smooth systems.

\section{Numerical simulations}

This section considers a numerical investigation of the system with discontinuous support. The proposed mathematical formulation is integrated with the aid of the Runge-Kutta-Fehlberg method. At first, it is considered an analysis related to the mathematical and numerical procedure here proposed. With this purpose, it is assumed parameters presented in Table 1. Figure 3 shows an example where the transition parameter $\eta=7.14 \times 10^{-3}$ and a numerical integration tolerance of $10^{-8}$ are considered. Under this condition, the system presents an evolution where part of the movement occurs without contact and the other part occurs with contact. Therefore, the system needs to pass for all subspaces previously defined. It is worthwhile to observe that, for this set of parameters, the system passes in all regions, i.e., the subspaces $\Gamma_{-}$and $\Gamma_{+}$, and the hyper-surfaces $\sum_{\alpha}$ and $\sum_{\beta}$.

Now, different narrow band thicknesses, $\eta$, are contemplated in order to highlight the previous conclusion. For this simulation, it is used the same parameters of Table 1 except $F_{0}=76.24 \mathrm{~s}^{-2}$ and $\omega=9.55 \mathrm{rad} / \mathrm{s}$, which is related to a chaotic response. The conclusions about this kind of motion are assured by experimental tests [13]. Figure 4 

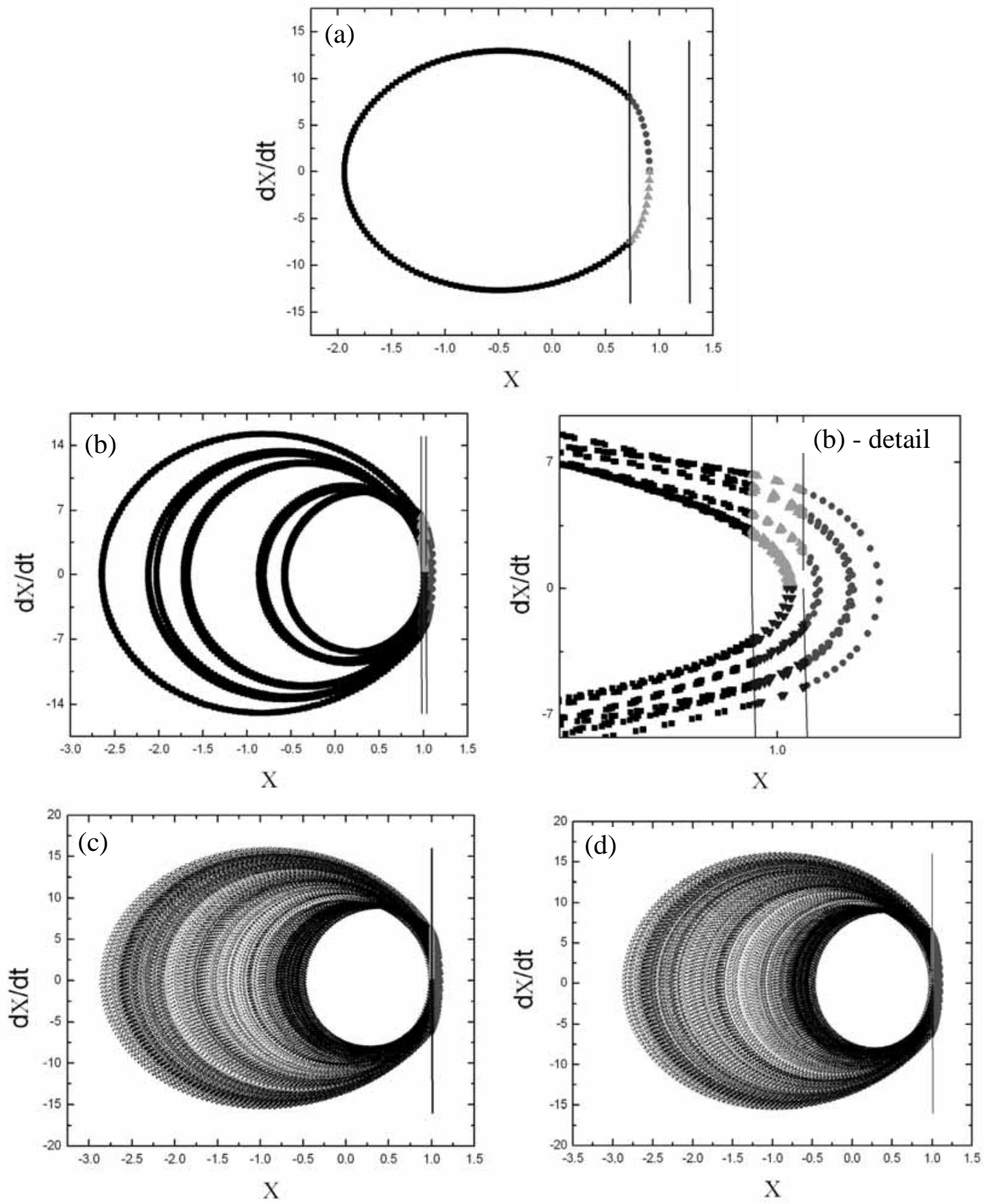

Fig. 4. Analysis of different narrow band thickness. (a) $\eta=2.8 \times 10^{-1}$; (b) $\eta=2.8 \times 10^{-2}$; (c) $\eta=2.8 \times 10^{-3}$; (d) $\eta=2.8 \times 10^{-4}$.

shows numerical simulations for $\eta=2.8 \times 10^{-1}, \eta=2.8 \times 10^{-2}, \eta=2.8 \times 10^{-3}$ and $\eta=2.8 \times 10^{-4}$. Notice that different qualitative responses are obtained due to the improper choice of the parameter $\eta$. When, $\eta=2.8 \times$ $10^{-1}$, the system does not pass to the region $\Gamma_{+}$since this value is not small enough. The decrease for a value $\eta=2.8 \times 10^{-2}$, still presenting numerical problems due to the same reason (observe the enlargement of this region showing an orbit that does not reach the region $\Gamma_{+}$). On the other hand, when the thickness of the narrow band is smaller than $\eta=2.8 \times 10^{-3}$, the system passes for all four regions, obtaining the expected result. Figure 4 presents simulations for $\eta=2.8 \times 10^{-3}$ and $\eta=2.8 \times 10^{-4}$ showing the same result. Based on this conclusion, it 
Table 2

System parameters

\begin{tabular}{ccccc}
\hline$\omega_{0}(\mathrm{rad} / \mathrm{s})$ & $\xi\left(\mathrm{s}^{-1}\right)$ & $\xi_{s}\left(\mathrm{~s}^{-1}\right)$ & $F_{0}\left(\mathrm{~s}^{-2}\right)$ & $\omega(\mathrm{rad} / \mathrm{s})$ \\
\hline 1 & 0.05 & 0.30 & 0.125 & 1 \\
\hline
\end{tabular}

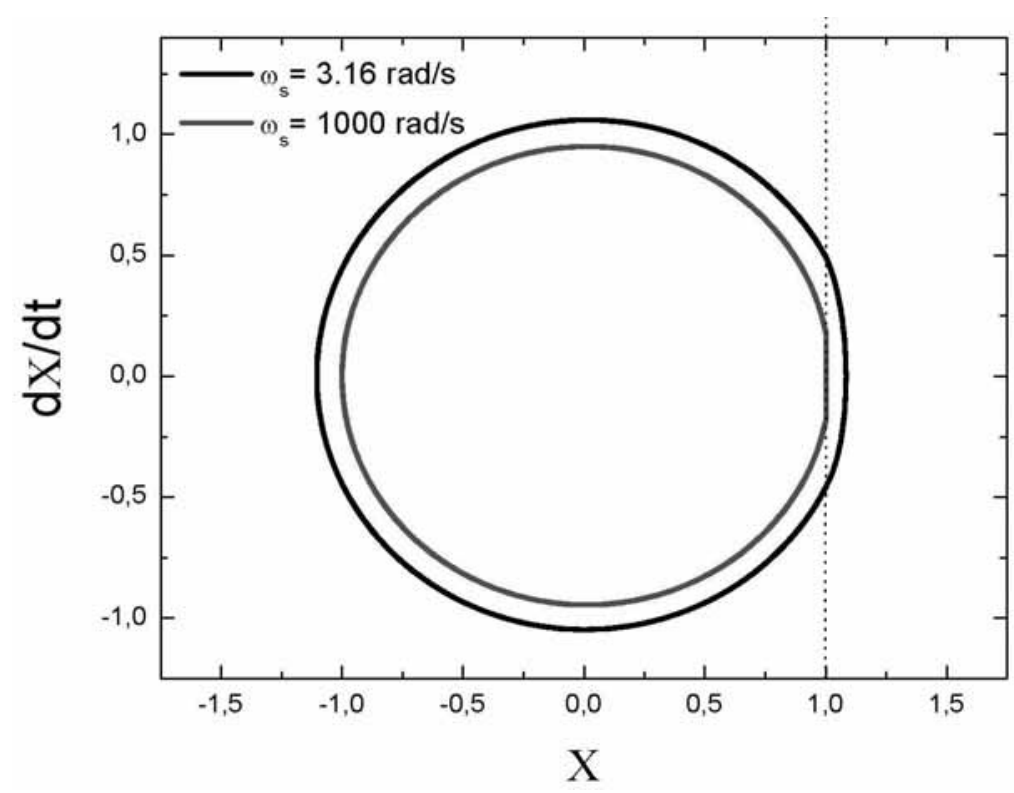

Fig. 5. Response for different support stiffness.

is assumed $\hat{\eta}=\eta g=10^{-5}$ as a reference value for all simulations. Actually, this value shown to be effective for all simulations here performed.

At this point, it is carried out an analysis of different kinds of support that may be related to distinct physical problems. Therefore, it is interesting to observe if the numerical procedure here proposed is able to analyze systems with high values of stiffness. This kind of response may be related to impact problems, for example. With this aim, it is assumed the system parameters presented in Table 2 , and also $\eta=2.5 \times 10^{-6}$. Figure 5 shows system response for different values of support stiffness. Notice that the numerical procedure does not present problems even when high values of support stiffness are considered.

Frequency domain analysis is now in focus considering the resonance curve of the system with discontinuous support (Fig. 6). These results are obtained by considering two different values of the support stiffness: $\omega_{s}=$ $3.16 \mathrm{rad} / \mathrm{s}$ (Fig. 6a) and $\omega_{s}=7.07 \mathrm{rad} / \mathrm{s}$ (Fig. 6b). The resonance curve has a typical characteristic of nonlinear systems, presenting dynamical jumps. Besides, it presents a discontinuity related to the contact with the support, caused by the hardening when contact occurs. Observing these curves, it is noticeable the frequency $\omega_{a}$ that is related to the contact event, and also the frequencies $\omega_{b}$ and $\omega_{c}$, which are associated with jumps. Notice that the hardening increase, associated with great support stiffness (Fig. 6b), tends to enlarge the effect of dynamical jumps.

In order to analyze dynamical jumps, different situations are now considered. When $\omega_{b}<\omega<\omega_{c}$ a bifurcation is observed and three periodic solution or attractors may coexist: two stable solutions and an unstable one [5,6]. The analysis of basins of attraction can be useful to observe this attractors coexistence. Figure 7 shows the evolution of the basins of attraction for $\omega_{s}=3.16 \mathrm{rad} / \mathrm{s}$ under increasing frequency $\omega$. In each case, it is observed two stable coexisting periodic attractors, represented by two different colors (dark and light). In Fig. 7(a), the excitation frequency is $\omega=1.10 \mathrm{rad} / \mathrm{s}$, while in Fig. 7(b) is $\omega=1.18 \mathrm{rad} / \mathrm{s}$. The light color basin of attraction represents a behavior without contact, while the dark basin of attraction is associated with a behavior with contact. Each one of these behaviors can be observed in the phase space presented in Fig. 8, obtained for $\omega=1.10 \mathrm{rad} / \mathrm{s}$. Initially, when $\omega=\omega_{b}$, all initial conditions lead to non-impact attractor (dark color). After that, the light attractor increases 
Table 3

System parameters

\begin{tabular}{ccccc}
\hline$\omega_{0}(\mathrm{rad} / \mathrm{s})$ & $\omega_{s}(\mathrm{rad} / \mathrm{s})$ & $\xi\left(\mathrm{s}^{-1}\right)$ & $\xi_{s}\left(\mathrm{~s}^{-1}\right)$ & $F_{0}\left(\mathrm{~s}^{-2}\right)$ \\
\hline 1 & 17.32 & 0.05 & 0.60 & 0.3125 \\
\hline
\end{tabular}

Table 4

System parameters

\begin{tabular}{ccccc}
\hline$\omega_{0}(\mathrm{rad} / \mathrm{s})$ & $\omega_{s}(\mathrm{rad} / \mathrm{s})$ & $\xi\left(\mathrm{s}^{-1}\right)$ & $\xi\left(\mathrm{s}^{-1}\right)$ & $\omega(\mathrm{rad} / \mathrm{s})$ \\
\hline 1 & 31.6 & 0.05 & 0.60 & 0.9 \\
\hline
\end{tabular}
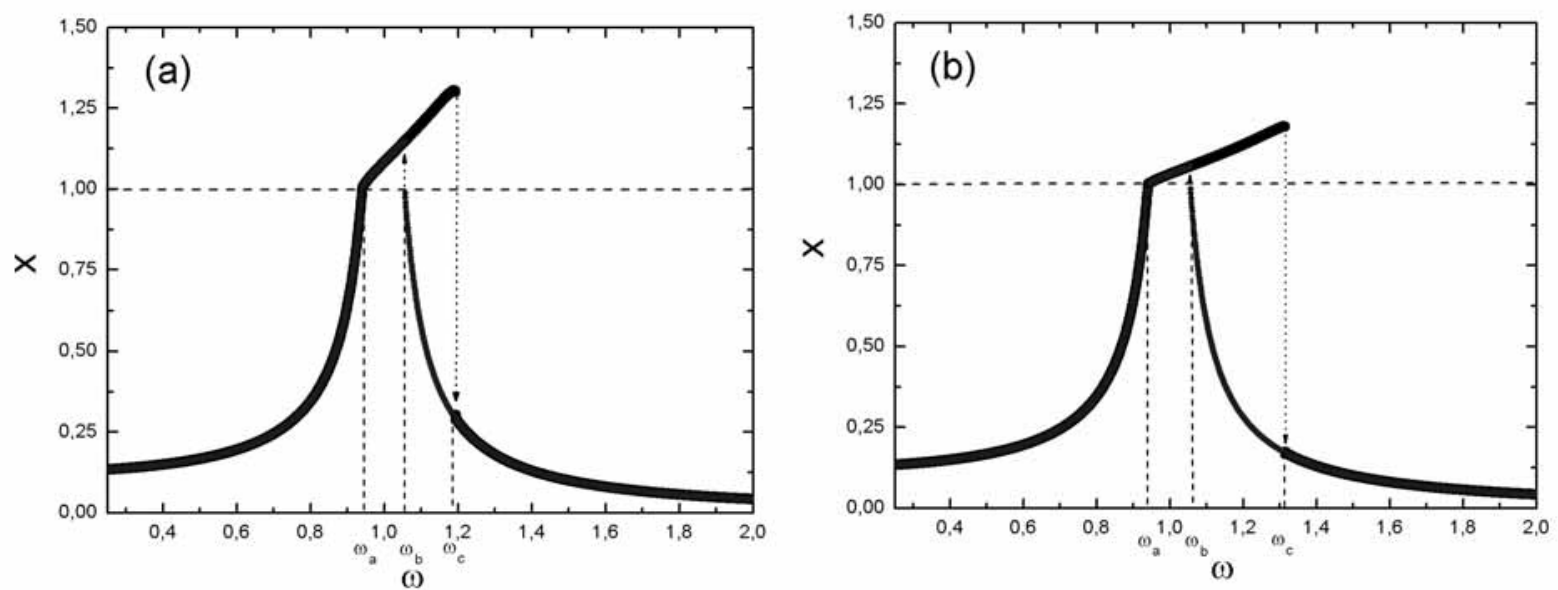

Fig. 6. Frequency domain analysis.
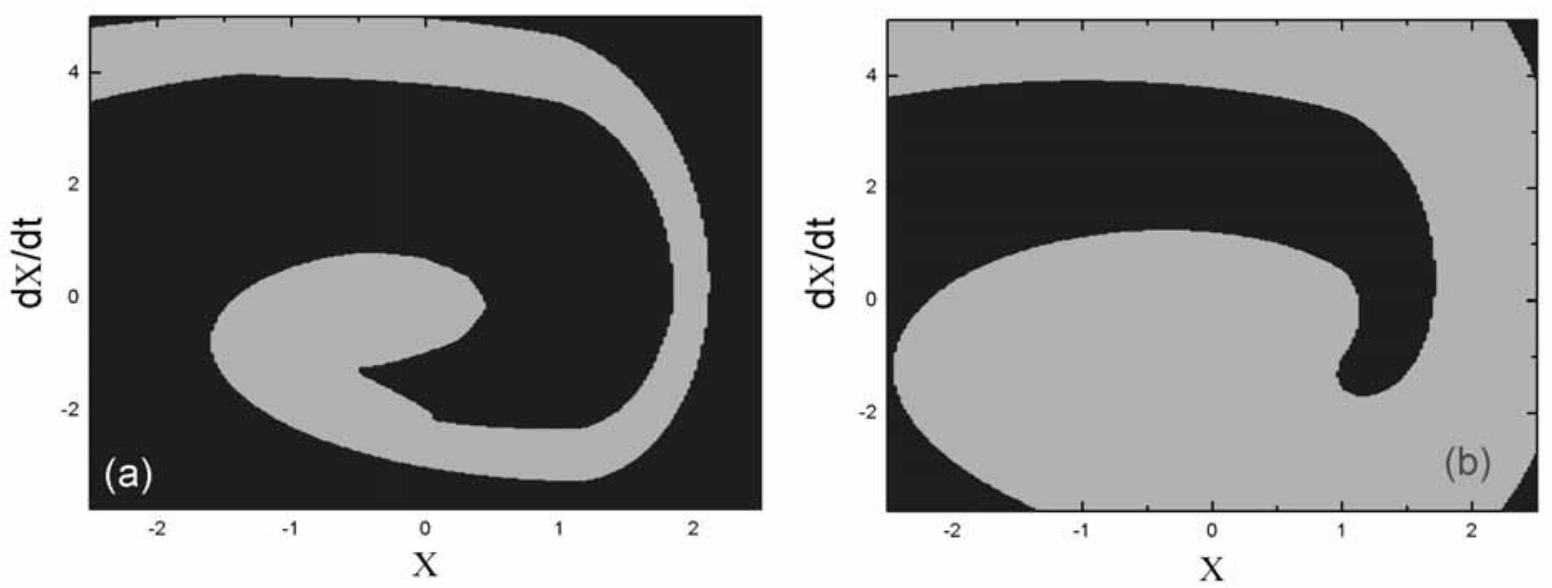

Fig. 7. Basins of attraction for different frequencies. (a) $\omega=1.10 \mathrm{rad} / \mathrm{s}$; (b) $\omega=1.18 \mathrm{rad} / \mathrm{s}$.

its size when excitation frequency is increased. The dynamical jump, when $\omega=\omega_{c}$, is represented when all initial conditions lead to an impact basin of attraction (light color).

The dynamical analysis of the discontinuous system here discussed shows that when the response has small amplitudes, there is no contact, and therefore a linear response is expected. This linear response is represented by an ellipse in the phase space. By changing the system forcing characteristics, the elliptical trajectory tends to grow until it reaches the support, presenting contact. The situation where the phase space ellipse touches lightly the boundary, is called grazing orbit. Under this condition, the mass grazes the support with zero velocity. Afterwards, a further 


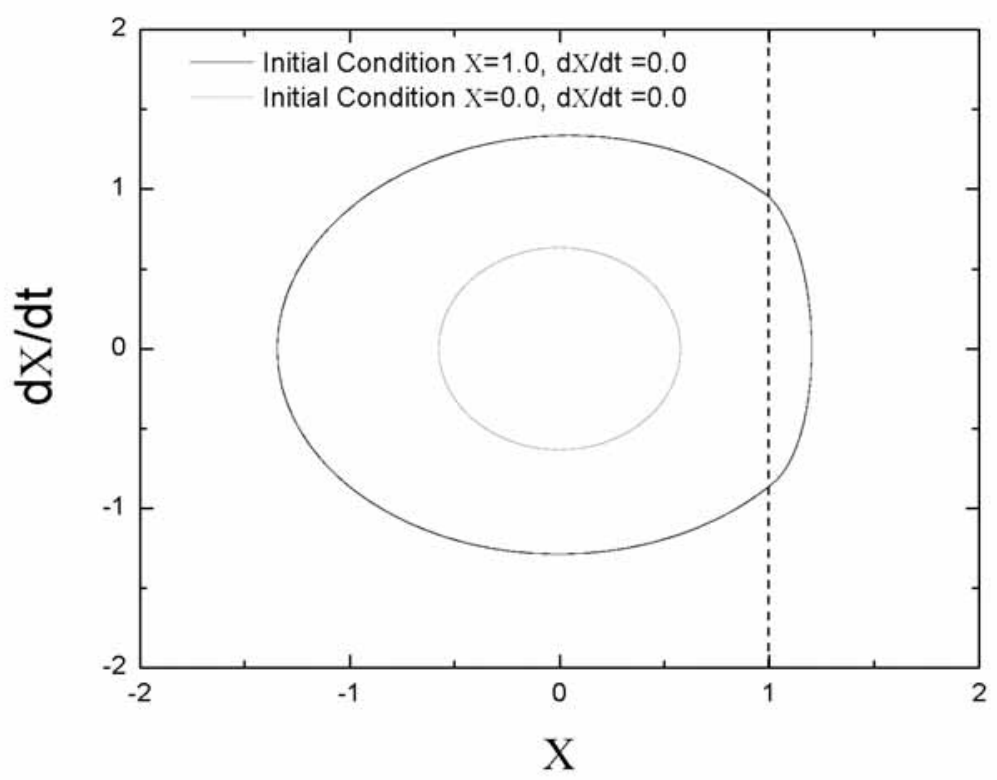

Fig. 8. Coexisting periodic attractors.
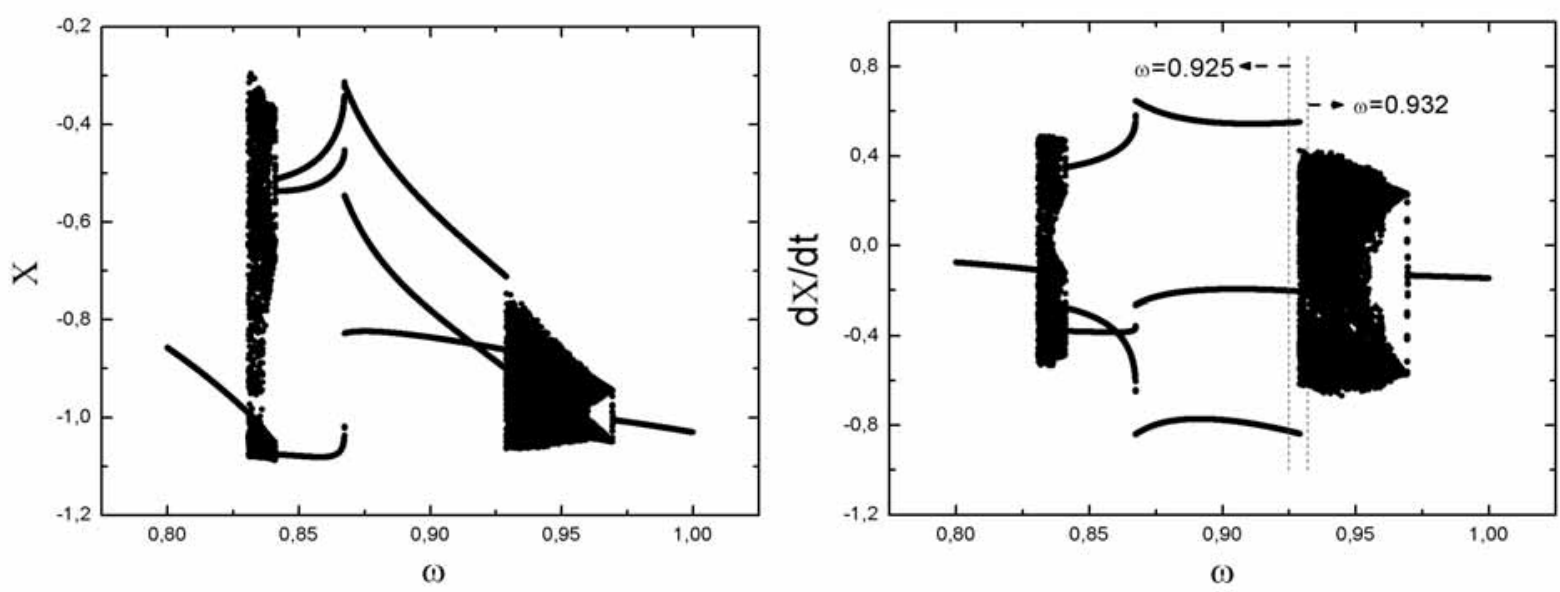

Fig. 9. Bifurcation diagram.

incremental change to the excitation characteristics produces contact behavior that is not easily predicted. For some combination of system parameters, the response may proceed abruptly into chaos. Higher-order periodic response may interrupt the chaotic regime as the forcing characteristics continue to change. On the other hand, the grazing orbit may be replaced directly by a stable periodic response. This unpredictability related to grazing bifurcations constitutes a typical behavior of non-smooth systems that does not appear in smooth systems [2,10-12,14].

The single-degree of freedom reported in this article allows one to show the grazing phenomenon and also different kinds of motion related to this. On this basis, it is considered parameters presented in Table 3 , with $\eta=6.25 \times$ $10^{-6}$. Figure 9 shows bifurcation diagram where the control parameter is the excitation frequency. This diagram gives an indication that the motion quickly moves into a chaotic region, characterizing a grazing contact. The phase space related to $\omega=0.91 \mathrm{rad} / \mathrm{s}$, is shown in Fig. 10(a), where it can be observed a period-3 orbit, in the imminence of grazing contact. By promoting a small increase in the forcing frequency, $\omega=0.92 \mathrm{rad} / \mathrm{s}$, the response becomes chaotic, as shown in the Poincaré map of the Fig. 10(b). Notice that by promoting small increases in the frequency 

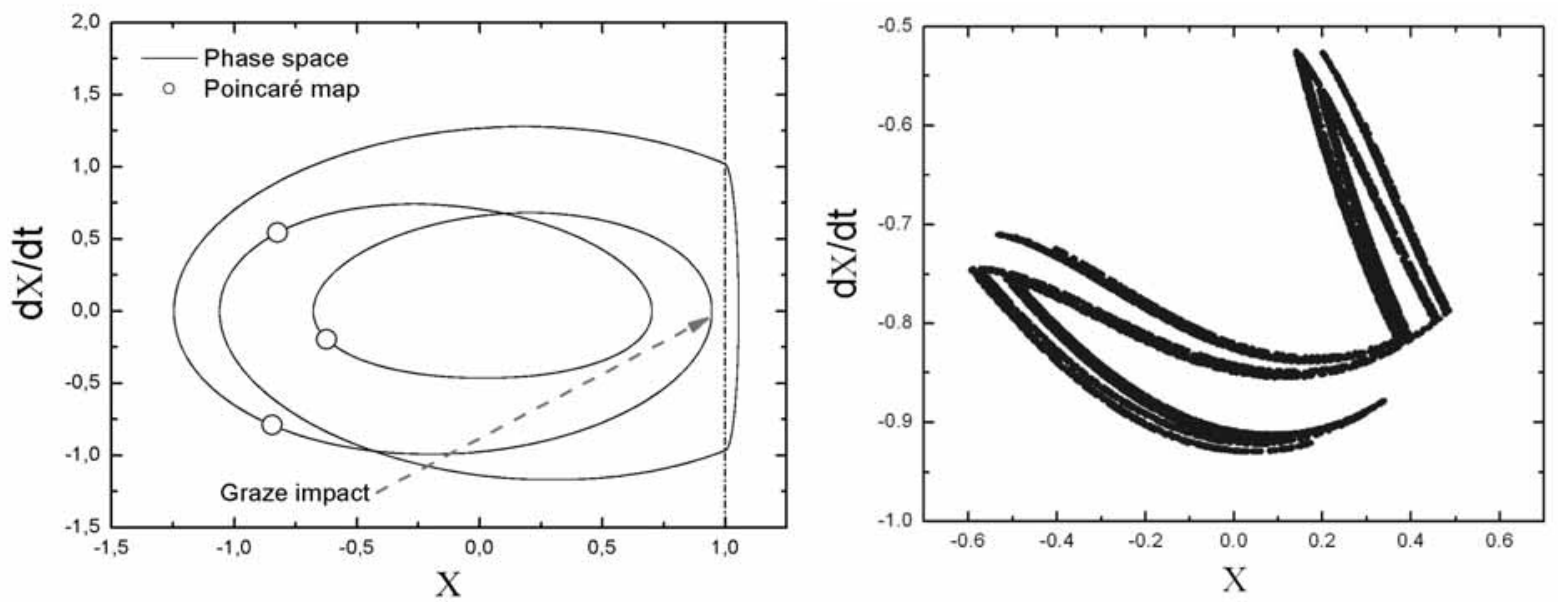

Fig. 10. Responses near the grazing orbit.
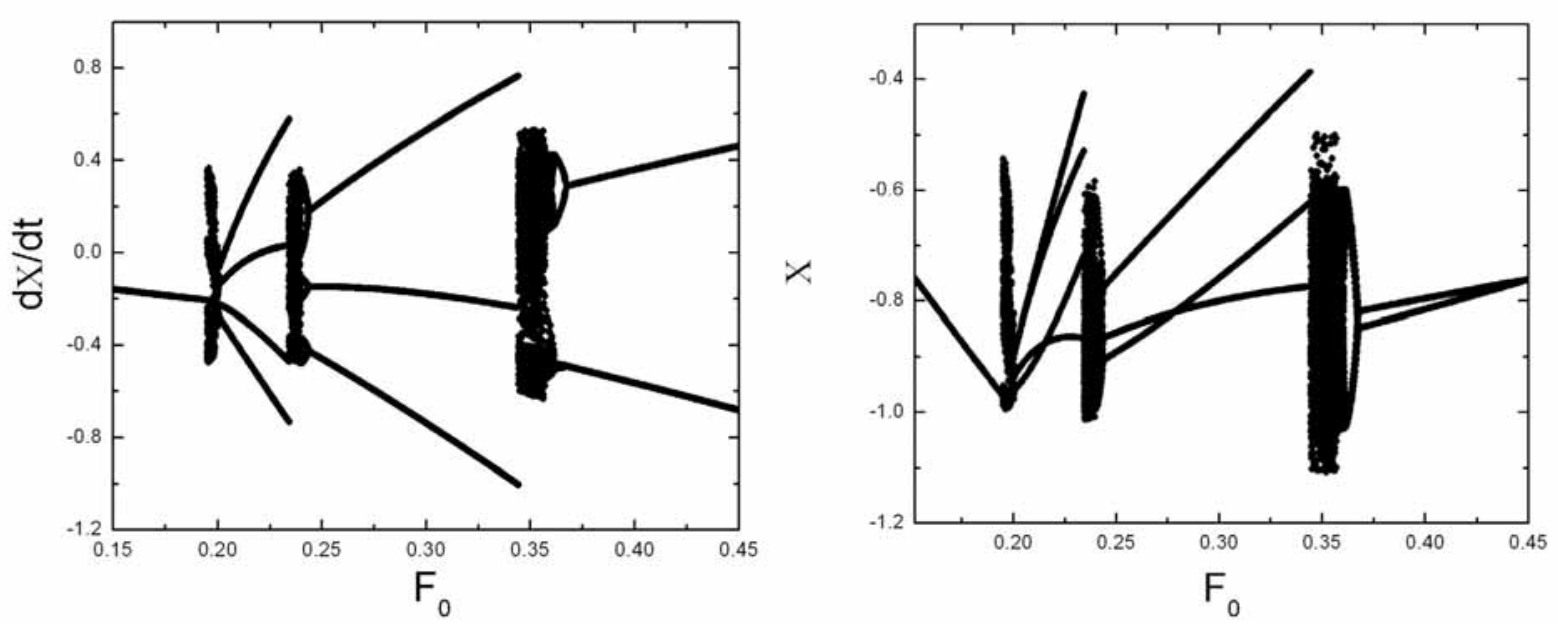

Fig. 11. Bifurcation diagram.

value near the grazing contact, the system reaches the support, presenting contact, and the response becomes irregular over a range of excitation frequency.

Now, a different bifurcation diagram is focused on, considering the forcing amplitude $F_{0}$ as a control parameter (Fig. 11). Table 4 presents the other parameters used on this analysis, together with $\eta=5 \times 10^{-6}$. Notice that, after a region related to periodic behaviors, chaotic regions appear. Once again, grazing orbit occurs just before the birth of the chaotic attractor. Phase spaces and Poincaré map of period-4 $\left(F_{0}=0.225 \mathrm{~s}^{-2}\right)$, chaotic $\left(F_{0}=0.350 \mathrm{~s}^{-2}\right)$ and period-2 $\left(F_{0}=0.400 \mathrm{~s}^{-2}\right)$ are presented in Fig. 12(a,b,c), respectively.

\section{Conclusions}

The objective of this research effort is the numerical investigation of non-smooth systems from a smoothened system. A single-degree of freedom oscillator with discontinuous support is analyzed as an application of this kind of system. A smoothened switch model is employed to model the system as four different sets of ordinary differential equations. Therefore, the state space is split into subspaces, and finite regions are used to define transitions among them. This procedure is useful for numerical simulations and seems to be effective in order to perform the integration 

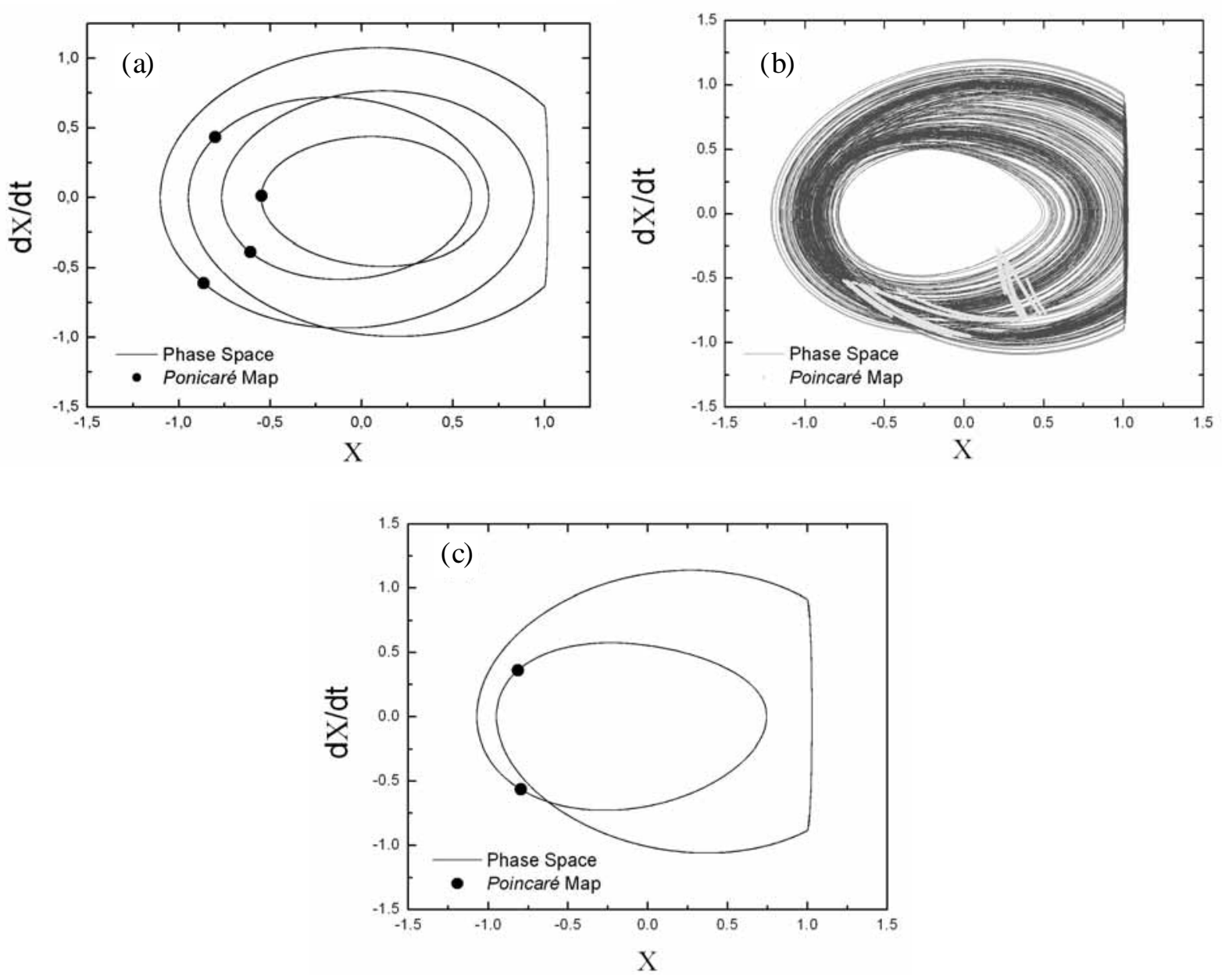

Fig. 12. Responses near the grazing orbit. (a) Period-4 $\left(F_{0}=0.225 \mathrm{~s}^{-2}\right)$; (b) Chaotic $\left(F_{0}=0.350 \mathrm{~s}^{-2}\right)$; (c) Period-2 $\left(F_{0}=0.400 \mathrm{~s}^{-2}\right)$.

of non-smooth systems. Numerical investigations are carried out allowing the analysis of different aspects related to the system dynamics. In general, the smoothened method presents stable behavior being more efficient than the direct integration of non-smooth system, allowing the use of larger time steps. Concerning the system behavior, its dynamics is rich, presenting dynamical jumps, bifurcations and chaos. The grazing bifurcation that causes abruptly changes in the system response reveals to be an important characteristic of this kind of system. Finally, the authors believe that the proposed procedure may be useful for the analysis of other non-smooth systems.

\section{Acknowledgement}

The authors would like to acknowledge the support of the Brazilian Research Council (CNPq).

\section{References}

[1] U. Andreaus and P. Casini, Dynamics of friction oscillators excited by a moving base and/or driving force, Journal of Sound and Vibration 245(4) (2001), 685-699.

[2] F. Casas, W. Chin, C. Grebogi and E. Ott, Universal grazing bifurcation in impact oscillators, Physical Review E 53 (1996), $134-139$. 
[3] A.F. Filippov, Differential equations with discontinuous right-hand side' American Mathematical Society Translations 42(Series 2) (1968), 199-231.

[4] N. Hinrichs, M. Oestreich and K. Popp, On the modelling of friction oscillators, Journal of Sound and Vibration 216(3) (1998), 435-459.

[5] R.I. Leine and D.H. van Campen, Discontinuous bifurcations of periodic solutions, Mathematical and Computer Modelling 36 (2002), 259-273.

[6] R.I. Leine and D.H. van Campen, Discontinuous fold bifurcation in mechanical systems, Archive of Applied Mechanics 72 (2002), 138-146.

[7] R.I. Leine and D.H. van Campen, Stick-slip vibrations induced by alternate friction models, Nonlinear Dynamics 16(1) (1998), 41-51.

[8] R.I. Leine, D.H. van Campen and B.L. van de Vrande, Bifurcations in nonlinear discontinuous systems, Nonlinear Dynamics 23 (2000), $105-164$.

[9] R.I. Leine, Bifurcations in Discontinuous Mechanical Systems of Filippov-Type, Ph.D. Thesis, Technische Universiteit Eindhoven, 2000.

[10] A.B. Nordmark, Non-periodic motion caused by grazing incidence in an impact oscillator, Journal of Sound and Vibration 145 (1991), 279-297.

[11] H.E. Nusse, E. Ott and J.A. Yorke, Border-collision bifurcation: An explanation for observed phenomena, Physical Review E 49 (1994), 1073-1076.

[12] E. Pavlovskaia, M. Wiercigroch and C. Grebogi, Modeling of an impact system with a drift, Physical Review E $64056224,2001$.

[13] M.A. Savi, S. Divenyi, L.F.P. Franca and H.I. Weber, Numerical and Experimental Investigations of the Nonlinear Dynamics and Chaos in Non-Smooth Systems with Discontinuous Support, COBEM 2005 - 18th International Congress of Mechanical Engineering, November 6-11, 2005, Ouro Preto.

[14] L.N. Virgin and C.J. Begley, Grazing bifurcations and basins of attraction in an impact-friction oscillator, Physica D 130 (1999), $43-57$.

[15] M. Wiercigroch, Modelling of dynamical systems with motion dependent discontinuities, Chaos, Solitons and Fractals 11 (2000), 24292442. 

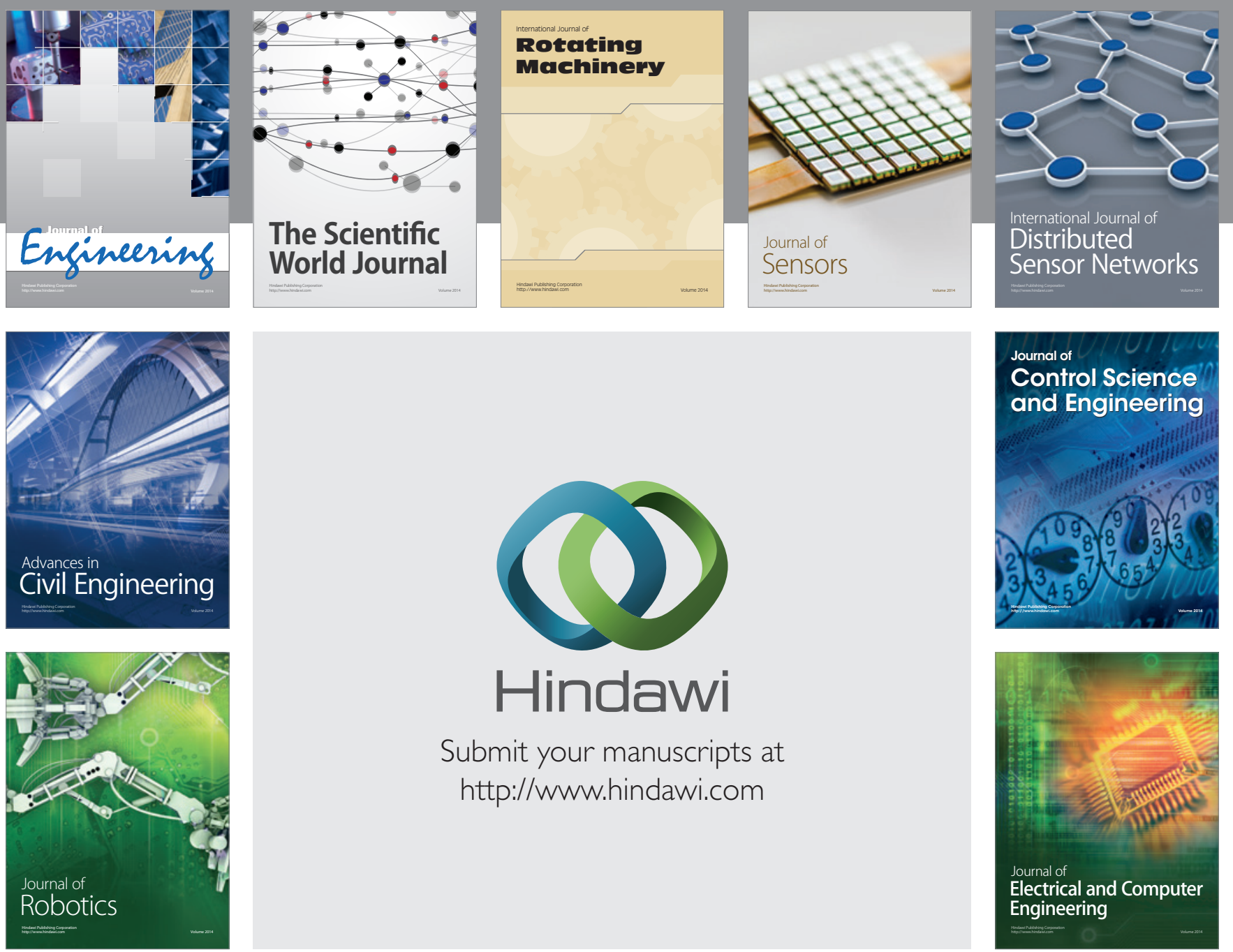

Submit your manuscripts at

http://www.hindawi.com
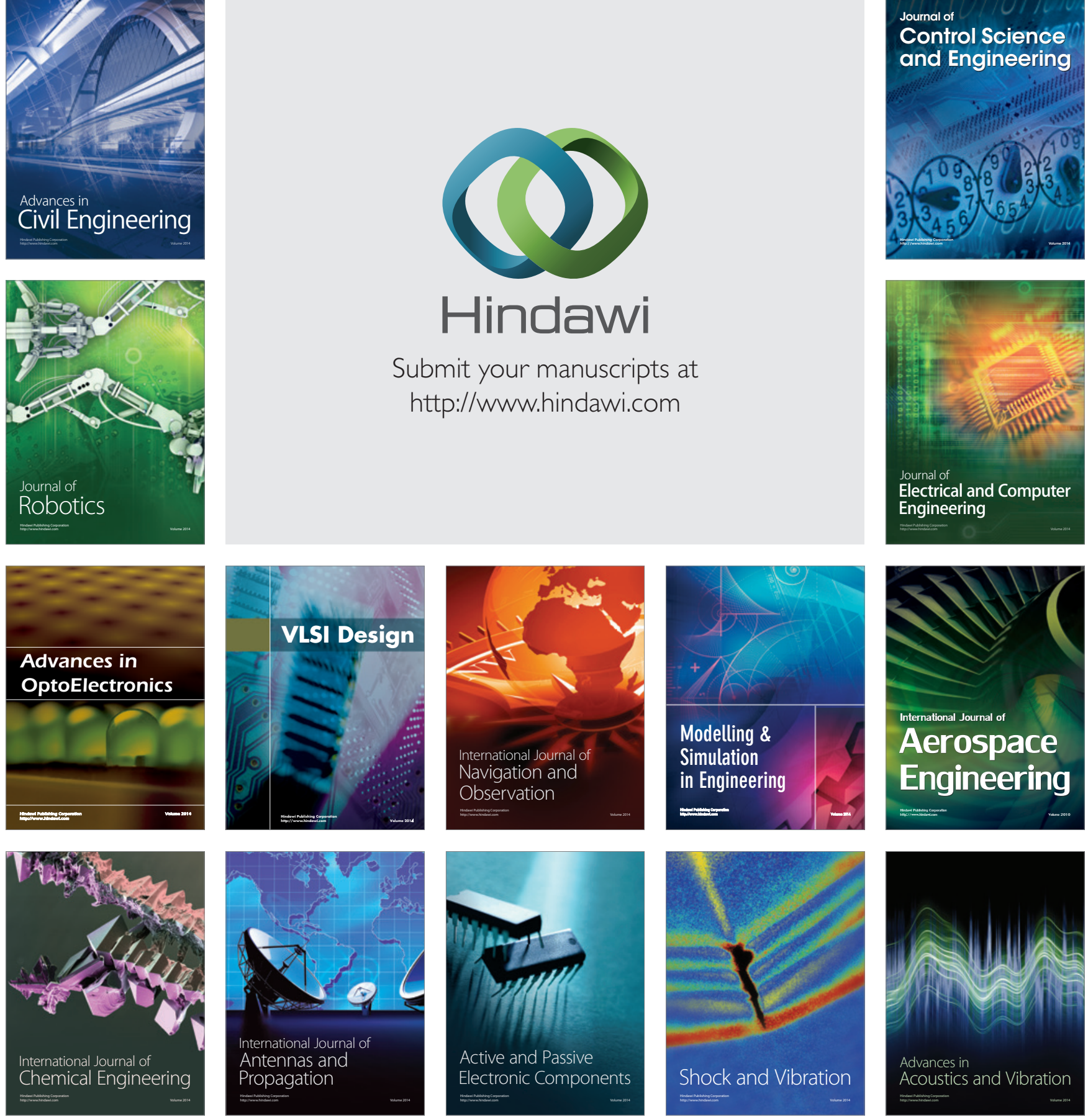